\title{
Rastreamento do câncer do colo do útero no Estado do Maranhão, Brasil
}

\author{
Cervical cancer screening in the State of Maranhão, Brazil
}

\author{
Diego Salvador Muniz da Silva ${ }^{1}$ \\ Ana Maria Nogueira Silva ${ }^{2}$ \\ Luciane Maria Oliveira Brito ${ }^{1}$ \\ Sinara Regina Lisboa Gomes ${ }^{1}$ \\ Maria do Desterro Soares Brandão Nascimento ${ }^{1}$ \\ Maria Bethânia da Costa Chein ${ }^{1}$
}

${ }^{1}$ Universidade Federal do Maranhão. Pç. Gonçalves Dias $21 / 2^{\circ}$, Centro. 65.020-240 São Luís MA Brasil.

diegosalvador27@hotmail.com

${ }^{2}$ Secretaria do Estado de

Saúde do Maranhão.

\begin{abstract}
The scope of the study was to analyze the screening for cervical cancer in the state of Maranhão using secondary data from the Cervical Cancer Information System (SISCOLO). A retrospective descriptive study was conducted using secondary data from 139505 cytopathology exams recorded in SISCOLO in Maranhão in 2011. The variables: age, education, adequacy of material, represented epithelia, microbiology and cellular changes detected in Pap smear tests were selected. The age group between 25 to 29 years and incomplete basic schooling were the most frequent findings. The most common microbiological agents detected were bacilli (52.8\%), cocci (45.5\%) and Lactobacillus sp (32.6\%). Inflammation was the most common benign cellular alteration (86.3\%). Low-grade intraepithelial lesions were the most prevalent atypical findings in squamous cells (0.6\%), followed by high-grade squamous intraepithelial lesions (0.2\%). Squamous cell carcinoma was observed in $0.003 \%$ and $0.006 \%$ for adenocarcinoma. SISCOLO proved a useful tool for studying aspects related to cancer screening of the cervix, which can orient actions to reduce the incidence and mortality from this cancer.
\end{abstract}

Key words Cervical cancer, Screening programs, Epidemiology, SISCOLO
Resumo O objetivo do estudo foi analisar o rastreamento do câncer do colo do útero no Maranhão, através dos dados do Sistema de Informação do Câncer do Colo do Útero (Siscolo). Foi realizado um estudo descritivo retrospectivo, com dados secundários dos 139.505 exames citopatológicos registrados no Siscolo do Maranhão, no ano de 2011. As variáveis: idade, escolaridade, adequabilidade do material, epitélios representados, microbiologia e alterações celulares detectadas ao exame citopatológico foram selecionadas. A faixa etária entre 25 a 29 anos e o ensino fundamental incompleto foram os mais frequentes. Os agentes microbiológicos mais comuns foram os bacilos $(52,8 \%), \operatorname{cocos}(45,5 \%)$ e Lactobacillus $\mathrm{sp}$ (32,6\%). A inflamação foi a alteração celular benigna mais frequente (86,3\%). As lesões intraepiteliais de baixo grau foram as atipias em células escamosas mais prevalentes (0,6\%), seguidas pela lesão intraepitelial de alto grau (0,2\%). O carcinoma epidermoide foi observado em $0,003 \%$ e o adenocarcinoma em 0,006\%. O Siscolo revelouse uma ferramenta útil para conhecer aspectos relacionados ao rastreamento do câncer do colo uterino, o que poderá guiar ações para reduzir a incidência e mortalidade por este câncer.

Palavras-chave Câncer do colo do útero, Programas de rastreamento, Epidemiologia, Siscolo 


\section{Introdução}

O câncer do colo do útero é um importante problema de saúde pública mundial. Estima-se o surgimento de aproximadamente 529 mil casos novos desse câncer no mundo, e para o Brasil espera-se 17.540 casos novos, sendo 780 localizados no Estado do Maranhão. Este câncer configura-se como o terceiro tipo mais frequente no sexo feminino, superado apenas pelos de pele não melanoma e mama ${ }^{1}$.

De evolução lenta, a história natural do câncer do colo do útero é descrita como uma afecção inicialmente de caráter benigno que sofre transformações intraepiteliais progressivas (duração média de 10 a 20 anos) e pode evoluir para um carcinoma invasor ${ }^{2}$. Por levar muitos anos para se desenvolver, é considerado raro em mulheres até 30 anos e sua incidência aumenta progressivamente até ter seu pico na faixa de 45 a 50 anos de idade ${ }^{3}$.

Sua incidência resulta da exposição das mulheres a fatores de risco e da eficiência dos programas de rastreamento 4 . Em 99,7\% dos casos, o HPV (papilomavírus humano) está relacionado ao câncer do colo do útero ${ }^{5,6}$. A infecção persistente pelos subtipos oncogênicos HPV-16 e HPV-18 originam cerca de $70 \%$ dos casos de câncer cervical invasor?

Além dos aspectos relacionados à infecção pelo HPV (subtipo, carga viral, infecção única ou múltipla), o tabagismo, a multiplicidade de parceiros sexuais, o uso de contraceptivos orais, múltiplos partos, baixa ingestão de vitaminas, iniciação sexual precoce e a coinfeccção por agentes infecciosos como o Vírus da Imunodeficiência Humana (HIV) e Chlamydia trachomatis se constituem outros fatores de risco para o desenvolvimento dessa patologia ${ }^{1,8,9}$.

O Ministério da Saúde (MS) recomenda o início do rastreamento para o câncer do colo uterino aos 25 anos de idade para as mulheres que já iniciaram atividade sexual. O intervalo entre os exames deve ser de três anos, após dois exames negativos, com intervalo anual. Os exames devem seguir até os 64 anos e serem interrompidos quando, após esta idade, as mulheres tiverem pelo menos dois exames negativos consecutivos nos últimos cinco anos ${ }^{3}$.

O diagnóstico precoce realizado por meio do exame preventivo (exame de Papanicolaou ou citopatológico) associado ao tratamento das lesões precursoras são fundamentais para prevenção e redução da mortalidade por este tipo de câncer ${ }^{3}$.
Devido à importância deste câncer, no ano de 1998 o MS instituiu o Programa Nacional de Combate ao Câncer de Colo do Útero através da Portaria GM/MS no 3040/98 ${ }^{10}$, que contava com a adoção de estratégias para estruturação da rede assistencial, desenvolvimento do sistema de informações, estabelecimento de mecanismos para mobilização e captação de mulheres, assim como definição das competências nos três níveis de governo.

Após a transferência da coordenação do programa para o Instituto Nacional do Câncer (Portaria GM/MS no 788/99), o MS criou o Sistema de Informação do Câncer do Colo do Útero (Siscolo), um software utilizado para o fornecimento de dados sobre identificação da paciente, informações demográficas, epidemiológicas e dos exames citopatológicos e histopatológicos realizados no Sistema Único de Saúde (SUS) ${ }^{10,11}$.

O Siscolo aprimorou-se ao longo dos anos e, atualmente, consiste numa ferramenta fundamental para organização das ações de rastreamento, informações gerenciais e faturamento dos exames. O Sistema permite ainda obter o boletim de produção ambulatorial individualizado, registrar informações sobre as condutas diagnósticas e terapêuticas relativas aos exames positivos/alterados, selecionar amostras para monitoramento externo da qualidade dos exames e coletar dados para construção de indicadores ${ }^{11,12}$.

Diante do exposto e considerando a relevância do assunto, este estudo busca realizar uma análise descritiva do rastreamento do câncer do colo uterino de mulheres atendidas nas Unidades Básicas de Saúde do Estado do Maranhão, cujos resultados dos exames citopatológicos foram transferidos ao Siscolo. Para isso, identificou-se as características da população estudada e os principais achados citopatológicos tais como alterações celulares benignas, atipias em células escamosas e glandulares e os principais agentes microbiológicos presentes no colo do útero destas mulheres.

\section{Métodos}

Realizou-se estudo retrospectivo observacional com dados dos exames citopatológicos coletados nas Unidades Básicas de Saúde (UBS) e cadastrados no Siscolo do Estado do Maranhão, referente ao período de janeiro a dezembro de 2011. A coleta de dados realizou-se no Departamento de Atenção à Saúde da Mulher e na Sala de Situação da Secretaria Adjunta de Atenção Pri- 
mária em Saúde do Maranhão, Secretaria do Estado de Saúde do Maranhão (SES-MA).

Foram selecionadas as variáveis referentes à idade, grau de escolaridade, adequabilidade do material coletado, epitélios representados, microbiologia e alterações celulares. As idades foram categorizadas em faixas etárias pré-estabelecidas (abaixo de 25; 25 a 64 e 65 ou mais anos), priorizando-se a faixa de 25 a 64 anos conforme recomendação do Ministério da Saúde ${ }^{3}$. Também se optou pela descrição das faixas etárias em intervalos de cinco anos, para melhor análise. A adequabilidade do material celular foi classificada como satisfatória ou não ${ }^{13}$. Os epitélios representados foram identificados no laudo dos exames como escamoso, glandular e/ou metaplásico. Os agentes microbiológicos, alterações celulares benignas, atipias em células escamosas e atipias em células glandulares também foram determinados.

Os dados quantitativos foram transferidos e analisados no programa Epi-Info versão 3.4 .3 e Microsoft Excel 2007. As frequências absolutas e relativas foram representadas sob forma de Tabelas e gráficos.

O estudo foi autorizado pela Secretaria do Estado de Saúde do Maranhão. Em conformidade com a Resolução n¹96/96 e o Conselho Nacional de Saúde ${ }^{14}$, o presente estudo possui aprovação do Comitê de Ética em Pesquisa do Hospital Universitário da Universidade Federal do Maranhão. O Termo de Consentimento Livre e Esclarecido não foi necessário por se tratar de dados secundários.

\section{Resultados}

Durante o ano de 2011, foram registrados 139.505 exames citopatológicos realizados nas UBS do Maranhão. A maioria dos exames $(76,8 \%)$ era de mulheres com idade entre 25 a 64 anos, sendo a faixa etária de 25 a 29 anos a mais frequente (Tabela 1, Figura 1). O ensino fundamental incompleto foi o nível de escolaridade mais prevalente $(41,8 \%)$, entretanto esta variável foi ignorada ou não preenchida em $89,6 \%$ dos exames. (Tabela 1).

A caracterização sociodemográfica com dados de etnia/raça, estado civil e ocupação não foram possíveis analisar em virtude da não transferência destas informações ao Siscolo pelas UBS maranhenses.

Considerando-se a adequabilidade do material celular, 96,9\% apresentaram celularidade ade- quada, sendo consideradas satisfatórias. Conforme Tabela 2, o epitélio escamoso foi o mais representado nas amostras citopatológicas $(96,3 \%)$, seguido pelo glandular $(51,7 \%)$.

Os agentes microbiológicos mais frequentes no colo uterino foram os bacilos $(52,8 \%)$, os cocos (45,5\%) e os Lactobacillus sp (32,6\%) (Tabela 3).

Dentre as alterações celulares benignas (reativas ou reparativas), a inflamação foi o achado mais frequente $(86,3 \%)$. As lesões intraepiteliais de baixo grau (LSIL) foram as atipias em células escamosas mais frequentes, presente em 835 exames $(0,6 \%)$. O carcinoma epidermoide foi evidenciado em $0,003 \%$ e adenocarcinoma em 0,006\% (Tabela 4).

\section{Discussão}

O rastreamento do câncer do colo uterino é realizado periodicamente através do exame citopatológico, sendo a estratégia preventiva mais adotada no Brasil ${ }^{3}$ e no mundo ${ }^{15}$. Segundo recomendações do MS, o exame deve ser realizado prioritariamente em mulheres de 25 a 64 anos, pois se observa baixa incidência e mortalidade pelo câncer do colo do útero fora desta faixa etária ${ }^{3}$. Há alta convicção de que o benefício líquido do cumprimento dessa recomendação etária é substancial, pois além da baixa incidência do câncer do colo do útero em mulheres até 24 anos, a maioria destes casos é diagnosticada no estádio I sendo o rastreamento menos eficiente para detectá$\operatorname{los}^{3}$. Apesar das recomendações do MS, o presente estudo revelou um expressivo percentual

Tabela 1. População estudada segundo faixa etária e escolaridade. Maranhão, 2011.

\begin{tabular}{lrr}
\hline \multicolumn{1}{c}{ Variáveis } & \multicolumn{1}{c}{$\mathbf{n}$} & \multicolumn{1}{c}{$\%$} \\
\hline Faixa etária (anos) & & \\
$\quad$ Abaixo de 25 & 2.6433 & 18,9 \\
Entre 25 e 64 & 107.174 & 76,8 \\
Acima de 64 & 5.898 & 4,2 \\
Escolaridade & & \\
$\quad$ Nenhuma/ analfabeto & 1.758 & 12,2 \\
Fundamental incompleto & 6.051 & 41,8 \\
Fundamental completo & 3.364 & 23,3 \\
$\quad$ Nível Médio & 2.892 & 20 \\
$\quad$ Nível Superior & 390 & 2,7 \\
\hline
\end{tabular}

*A variável foi classificada como "Ignorado/sem informação" em $89,6 \%(n=125.050)$ dos casos.

Fonte: SES-MA. 


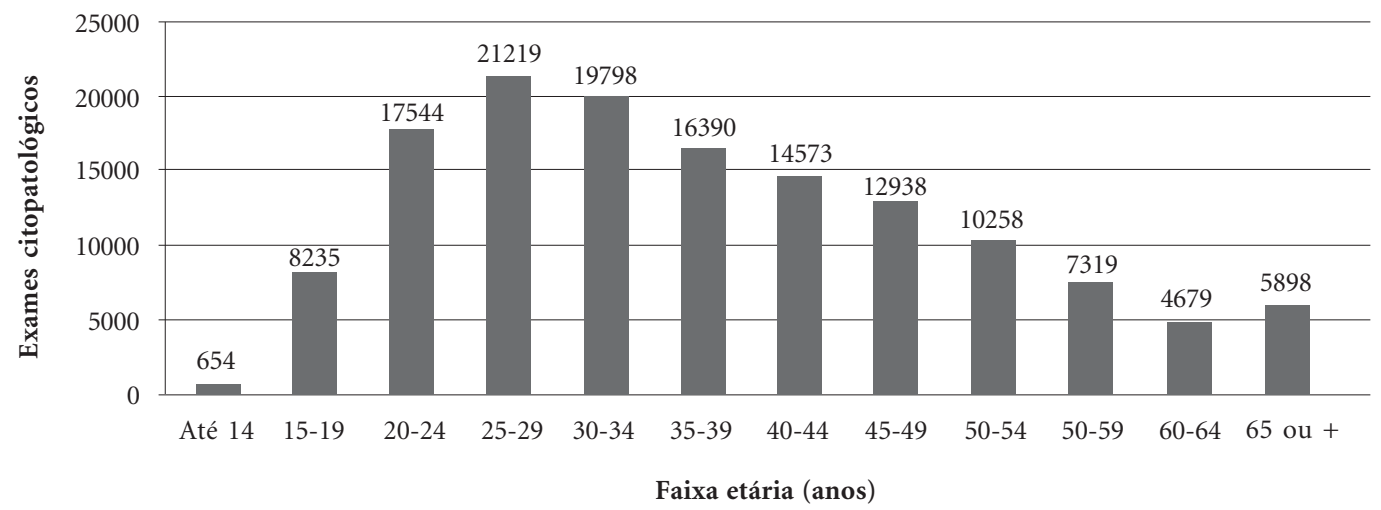

Figura 1. Distribuição dos exames citopatológicos conforme a faixa etária. Maranhão, 2011.

Fonte: SES-MA.

Tabela 2. Epitélios representados na amostra citopatológica da população estudada. Maranhão, 2011.

\begin{tabular}{lrc}
\hline \multicolumn{1}{c}{ Epitélios } & \multicolumn{1}{c}{$\mathbf{n}$} & \% \\
\hline Escamoso & 134.390 & 96,3 \\
Glandular & 72.134 & 51,7 \\
Metaplásico & 17.485 & 12,5
\end{tabular}

Fonte: SES-MA.

Tabela 3. Principais agentes microbiológicos ao exame citopatológico da população estudada. Maranhão, 2011.

\begin{tabular}{lrr}
\hline \multicolumn{1}{c}{ Microbiologia } & \multicolumn{1}{c}{$\mathbf{n}$} & \multicolumn{1}{c}{$\%$} \\
\hline Bacilos & 73.610 & 52,8 \\
Cocos & 63.498 & 45,5 \\
Lactobacillus sp & 45.525 & 32,6 \\
Trichomonas vaginalis & 2.375 & 1,7 \\
Chlamydia sp & 53 & 0,04 \\
Efeitos citopático compatível & 35 & 0,02 \\
com vírus do grupo Herpes & & \\
Actinomyces sp & 14 & 0,01 \\
Outros & 2.167 & 1,5 \\
\hline
\end{tabular}

Fonte: SES-MA.

de realização do exame citopatológico fora da faixa etária preconizada $(23,2 \%)$, o que sugere um número significativo de procedimentos diagnósticos e terapêuticos desnecessários.
Tabela 4. Alterações celulares ao exame citopatológico da população estudada. Maranhão, 2011.

\begin{tabular}{lrr}
\hline \multicolumn{1}{c}{ Variáveis } & n & \% \\
\hline Alterações celulares benignas & & \\
$\quad$ Inflamação & 120.436 & 86,3 \\
Atrofia com inflamação & 10.172 & 7,3 \\
Metaplasia escamosa imatura & 3.969 & 2,8 \\
Radiação & 294 & 0,2 \\
Reparação & 235 & 0,2 \\
Outros & 8114 & 5,8 \\
Atipias em células escamosas & & \\
$\quad$ Lesão intraepitelial de baixo grau & 835 & 0,6 \\
Lesão intraepitelial de alto grau & 284 & 0,2 \\
Carcinoma epidermóide invasor & 5 & 0,003 \\
Atipias em células glandulares & & \\
$\quad$ Adenocarcinoma & 10 & 0,006 \\
\hline
\end{tabular}

Fonte: SES-MA.

A distribuição homogênea em colunas das faixas etárias do estudo apresentou pico de incidência na terceira e quarta década de vida, semelhante a outros estudos ${ }^{16-20}$. Observou-se que 654 exames citopatológicos foram realizados por mulheres com idade inferior aos 14 anos, apontando para o início precoce das relações sexuais, o que aumenta o risco do câncer cervical. Tal relação justifica-se, pois a zona de transformação do epitélio cervical é mais proliferativa durante a puberdade e adolescência (período vulnerável), sen- 
do principalmente susceptível a alterações que podem ser induzidas por agentes transmitidos, especialmente o HPV. Na adolescência há uma probabilidade maior desta infecção virótica se converter em um processo crônico, o que implicaria em um risco maior do desenvolvimento de câncer cervical ${ }^{17}$. Concomitantemente, observase a necessidade na implantação de programas de atenção voltados para as adolescentes ${ }^{21}$, a fim de diminuir o avanço progressivo de casos de lesões precursoras do carcinoma de cérvice uterina ${ }^{22}$.

A maioria das mulheres que realizaram os exames citopatológicos possuía baixo nível de escolaridade. Excluindo os exames ignorados/em branco, verifica-se que $41,8 \%$ possuíam primeiro grau incompleto. Thuler et al. ${ }^{23}$, em estudo observacional dos casos de câncer do colo do útero no Brasil, identificaram o ensino fundamental incompleto (49\%) como a escolaridade mais prevalente, sugerindo ser um fator associado para o desenvolvimento do câncer cervical, fato também semelhante ao encontrado em mulheres residentes de uma área coberta pelo Programa Saúde da Família em um município do Maranhão ${ }^{24} \mathrm{com}$ prevalência desta escolaridade em 51,2\%. Provavelmente esta associação é uma realidade sociodemográfica brasileira, em especial das mulheres que buscam atendimento no SUS.

Designa-se satisfatória, a amostra que apresente células em quantidade suficiente, bem distribuídas, fixadas e coradas, de tal modo que sua visualização permita uma conclusão diagnóstica. Neste critério não se considera a representatividade epitelial da amostra, sendo esta exclusivamente de competência do profissional que realiza a coleta. Por outro lado, a presença de células metaplásicas ou endocervicais, representativas da junção escamo-colunar, tem sido considerada indicador da qualidade do exame, pelo fato delas se originarem do local onde se situa a quase totalidade dos cânceres do colo do útero ${ }^{13}$.

No presente estudo, identificou a presença do epitélio escamoso em quase a totalidade dos exames (96,3\%). Clinicamente, para a boa qualidade do exame, espera-se que a representação dos epitélios glandulares e/ou metaplásicos seja pelo menos igual ao escamoso, a sua ausência é considerada normal somente nas mulheres submetidas à histerectomia. Entretanto, a soma destes epitélios $(64,2 \%)$ foi abaixo do desejado, o que pode ser um indício da má qualidade da coleta e/ ou fixação do material, provavelmente por despreparo teórico-prático dos profissionais para sua realização. Esta situação compromete a avaliação clínica, pois não propiciará às mulheres todos os benefícios do rastreamento do câncer do colo do útero ${ }^{13}$.

Campos et al. ${ }^{22}$ ao analisarem os resultados citológicos das amostras cérvico-vaginais coletadas em um município do Maranhão identificaram frequências aproximadas: bacilos 56,8\%, $\operatorname{cocos} 42,2 \%$ e lactobacilos 35,2\%. Devido à presença de grande variedade de bactérias no conteúdo cérvico-vaginal, estes agentes são normalmente detectados em mulheres assintomáticas, formando uma flora mista composta de cocos e bacilos, além dos lactobacilos.

Dentre as alterações celulares benignas (reativas ou reparativas), a inflamação foi o achado mais frequente dos exames citopatológicos, corroborando com outros estudos ${ }^{18,25}$. A inflamação é uma alteração celular epitelial comum no colo uterino e vagina, em virtude da ação de agentes físicos, os quais podem ser radioativos, mecânicos ou térmicos, e químicos como medicamentos abrasivos ou cáusticos, quimioterápicos e acidez vaginal sobre o epitélio glandular ${ }^{26}$. Vale ressaltar que o processo inflamatório intenso prejudica a qualidade da amostra, devendo ser realizado o tratamento e a nova coleta citológica após três meses. Havendo positividade na citologia subsequente, deve-se encaminhar a paciente à unidade de referência para colposcopia ${ }^{27}$.

Neste estudo, as LSIL foram verificadas em 0,6\% dos exames citopatológicos, prevalência inferior à nacional que foi de $0,8 \%$ em $2009^{28}$. Isto sugere subnotificação dos casos e/ou ausência destas informações no Siscolo, pois se espera uma prevalência superior à encontrada, por se tratar de uma patologia cuja epidemiologia regional é influenciada pelo baixo nível socioeconômico $^{29}$. A conduta para mulheres com este diagnóstico é repetir o exame em seis meses na UBS. Havendo repetição do diagnóstico, a colposcopia esta indicada em unidade de referência para avaliar a necessidade de biópsia dirigida para estudo histopatológico da área suspeita ${ }^{3}$.

O efeito citopático pelo HPV e a lesão intraepitelial cervical grau I compreendem as LSIL, e o método Papanicolaou detecta somente as alterações induzidas por HPV e não o vírus propriamente dito, possuindo baixa sensibilidade. Apenas métodos moleculares são capazes de identificar o vírus, como a captura híbrida e a reação em cadeia da polimerase ${ }^{30-32}$.

Com uma melhor compreensão da biologia de infecções pelo HPV, a vacina contra HPV tem sido uma ferramenta utilizada em vários países para prevenção do HPV e câncer do colo do útero $^{31,33}$. Sua incorporação no Programa Nacional 
de Imunizações permanece em discussão pelo MS e pode se tornar uma das ferramentas para o controle do câncer do colo do útero no Brasil, sobretudo em mulheres jovens. Pesquisadores franceses ao estudar a eficácia da vacina contra HPV para prevenir o câncer cervical concluíram que aumentar a cobertura vacinal em mulheres ou vacinar meninas antes dos 14 anos mostrou um melhor impacto sobre a incidência deste câncer. A vacinação em homens melhora apenas moderadamente o efeito sobre a incidência de câncer cervical, em comparação com as estratégias adotadas para a população feminina ${ }^{34}$.

A prevalência do diagnóstico citopatológico de adenocarcinoma no Brasil foi inferior a $0,01 \%$, dentre os exames realizados no ano de $2009^{28}$. As atipias em células glandulares deste estudo comportaram-se abaixo do esperado, apresentando uma baixa prevalência, assim como verificado em outros estudos ${ }^{18,22,25}$. Contudo, acredita-se que a subnotificação dos casos e/ou ausência destas informações no Siscolo também contribuíram para este baixo indicador. As pacientes acometidas com tais atipias devem ser encaminhadas para colposcopia na atenção secundária, com indicação de conização. Entretanto, se à colposcopia for observada alteração sugestiva de invasão, a biópsia deverá ser realizada. Caso o diagnóstico histopatológico confirme essa suspeita, a paciente deverá ser encaminhada para atenção terciária ${ }^{3}$.

Diante da análise dos dados do Siscolo, observaram-se dificuldades e limitações na realização deste estudo. O Siscolo não permite a correta identificação nem o número de mulheres examinadas, sendo útil apenas para quantificar os exames citopatológicos realizados e conhecer aspectos relacionados. Além disso, há uma porcentagem insuficiente de mulheres submetidas à coleta do citopatológico que, somado às grandes desigualdades regionais e sociais brasileiras, levam a valores muito díspares, o que dificulta o conhecimento preciso das reais taxas de captação e cobertura, essenciais ao acompanhamento das ações planejadas ${ }^{3,35}$.

Dentre as limitações do Siscolo, destaca-se a falta de controle sobre quem está fazendo os exames e o intervalo entre os mesmos, o que resulta no super-rastreamento de algumas mulheres em detrimento de outras 3 . Além disso, há coleta de citologias desnecessárias (em intervalos menores e para grupos etários não previstos pelo programa de rastreamento) que chegam a cifras de mais de $60 \%$ de todos os exames ${ }^{36}$. Cabe ainda citar outras limitações como a ausência de meca- nismos que garantam a obrigatoriedade na submissão de dados (sóciodemográficos, procedimentos e exames realizados anteriormente) no Sistema; a falta de acesso às informações dos exames de rastreamento nas UBS onde é realizada a coleta do material; e os dados existentes no Siscolo estarem restritos à população usuária do SUS, não englobando as mulheres que realizam os exames em serviços de saúde suplementar.

Visando o aprimoramento nas ações de rastreamento, o MS está desenvolvendo a versão web do Siscolo, que será integrada ao Sistema de Informação do Câncer (Siscan). Este sistema está vinculado ao Cadastro Nacional de Cartão de Saúde e possibilitará a identificação da mulher, além de acabar com a necessidade de transferência de arquivos entre as coordenações. As informações inseridas no Siscan estarão disponíveis em tempo real pela internet, possibilitando às UBS realizar exames de rastreamento segundo periodicidade e faixa etária recomendadas, acessar exames já realizados e cadastrar informações no módulo seguimento, entre outras funcionalidades ${ }^{37}$.

O Siscolo é uma ferramenta útil para conhecer aspectos relacionados ao rastreamento do câncer do colo uterino no Brasil. Contudo, há deficiências e limitações que necessitam ser corrigidas, além do fortalecimento do Siscolo (que deverá ser alcançado através da implantação do Siscan) e capacitações/qualificação profissional. Por meio dessas ações, e da implementação de Políticas Públicas destinadas à Saúde da Mulher e ao rastreamento do câncer de colo uterino no Brasil, espera-se melhorar os indicadores de saúde da população feminina, principalmente através da redução das taxas de incidência e mortalidade pelo câncer do colo uterino.

\section{Colaboradores}

DSM Silva participou da elaboração do artigo, além da concepção, coleta e análise de dados; AMN Silva participou da concepção, coleta e revisão final do artigo; LMO Brito participou da concepção e elaboração do artigo; SRL Gomes participou da revisão do artigo e análise crítica; MDSB Nascimento participou na redação final do artigo; MBC Chein participou da elaboração do artigo, além da concepção, delineamento, coleta e análise de dados. 


\section{Referências}

1. Brasil. Ministério da Saúde (MS). Instituto Nacional de Câncer (Inca). Estimativa 2012: incidência de câncer no Brasil. Rio de Janeiro: Inca; 2011.

2. Brasil. Ministério da Saúde (MS). Instituto Nacional de Câncer (Inca). Falando sobre câncer do colo do útero. Rio de Janeiro: Inca; 2002.

3. Brasil. Ministério da Saúde (MS). Instituto Nacional de Câncer (Inca). Diretrizes brasileiras para o rastreamento do câncer do colo do útero. Rio de Janeiro: Inca; 2011.

4. Feitosa TMP, Almeida RT. Perfil de produção do exame citopatológico para controle do câncer do colo do útero em Minas Gerais, Brasil, em 2002. Cad Saude Publica 2007; 23(4):907-917.

5. Walboomers JM, Jacobs MV, Manos MM, Bosch FX, Kummer JA, Shah KV, Snijders PJ, Peto J, Meijer CJ, Muñoz N. Human papillomavirus is a necessary cause of invasive cervical cancer worldwide. $J$ Pathol 1999; 189(1):12-19.

6. Brasil. Ministério da Saúde (MS). Instituto Nacional de Câncer (Inca). HPV: Perguntas e respostas mais frequentes. [Internet]. [cited 2012 Oct 28]. Available from: http://www.inca.gov.br/conteudo_view. asp?id $=327$

7. World Health Organization (WHO). ICO Information Centre on Human Papilloma Virus (HPV) and Cervical Cancer. Human papillomavirus and related cancers in Brazil. 2010. [Internet]. [cited $2010 \mathrm{Jul}$ 20]. Available from: www.who.int/hpvcentre

8. International collaboration of epidemiological studies of cervical cancer. Cervical carcinoma and sexual behavior: collaborative reanalysis of individual data on 15,461 women with cervical carcinoma and 29,164 women without cervical carcinoma from 21 epidemiological studies. Cancer Epidemiol Biomarkers Prev 2009; 18(4):1060-1069.

9. Munoz N, Bosch FX, de Sanjose S, Herrero R, Castellsague X, Shah KV, Snijders PJ, Meijer CJ; International Agency for Research on Cancer Multicenter Cervical Cancer Study Group. Epidemiologic classification of human papillomavirus types associated with cervical cancer. $N$ Engl J Med 2003; 348(6): 518-527.

10. Brasil. Ministério da Saúde(MS). Instituto Nacional de Câncer (Inca). Programa Nacional de Controle do Câncer do Colo do Útero e Programa Nacional de Controle do Câncer de Mama. Rio de Janeiro: Inca; 2010.

11. Brasil. Ministério da Saúde(MS). Instituto Nacional de Câncer (Inca). Sistema de informação do controle do câncer de mama (SISMAMA) e do câncer do útero (SISCOLO): manual gerencial. Rio de Janeiro: Inca; 2011.

12. Dias MBK, Gláucia J, Assis TM. Rastreamento do câncer de colo do útero no Brasil: análise de dados do Siscolo no período de 2002 a 2006. Epidemiol Serv Saúde 2010; 19(3):293-306.

13. Instituto Nacional de Câncer (Inca). CoordenaçãoGeral de Prevenção e Vigilância. Divisão de Detecção Precoce e Apoio à Organização de Rede. Nomenclatura Brasileira para Laudos Citopatológicos Cervicais. $3^{a}$ ed. Rio de Janeiro: Inca; 2012.
14. Brasil. Ministério da Saúde (MS). Fundação Nacional da Saúde Centro Nacional de Epidemiologia. Resolução no196/96 sobre pesquisa envolvendo seres humanos. Inf Epidemiol SUS 1996; 5(2):13-41.

15. World Health Organization (WHO). Programmes and projects. Cancer. Screening and early detection of cancer. 2010. [Internet]. [cited 2010 Nov 19]; Available from: http://www.who.int/cancer/ detection/cytologyscreen/en/index.html

16. Murata IMH, Gabrielloni MC, Schirmer J. Cobertura do Papanicolaou em Mulheres de 25 a 59 anos de Maringá - PR, Brasil. Rev Brasileira de Cancerologia 2012; 58(3):409-415.

17. Medeiros VCRD, Medeiros RC, Moraes LM, MenezesFilho JB, Ramos ESN, Saturnino ACRD. Câncer de Colo de Útero: Análise Epidemiológica e Citopatológica no Estado do Rio Grande do Norte. Rev Bras Anal Clin 2005; 37(4):227-231.

18. Terres AF, Machado NA, França FS, Ramos CDT, Haas P. Análise dos resultados de exames preventivos e de rastreamento de câncer de colo do útero realizados em uma clínica ginecológica particular no município de Curitiba, PR. Estud Biol 2009; 31(73/ 74/75):103-109.

19. Uchimura NS, Nakano K, Nakano LCG, Uchimura TT. Qualidade e desempenho das colpocitologias na prevenção de câncer de colo uterino. Rev Assoc Med Bras 2009; 55(5):569-574.

20. Oliveira MMHN, Silva AAM, Brito LMO, Coimbra LC. Cobertura e fatores associados à não realização do exame preventivo de Papanicolaou em São Luís, Maranhão. Rev Bras Epidemiol 2006; 9(3):325334.

21. Krogh E, Brito LMO, Silva DSM, Ferreira ECMF, Silva RA. Consulta ginecológica na adolescência: aspectos relacionados e motivações determinantes para sua realização. Rev Pesq Saúde 2012; 13(2):1116.

22. Campos RS, Silva IC, Silva LRL, Pinto MLS, Mochel EG. Prevenção do câncer de colo uterino: achados citológicos e microbiológicos em mulheres e uma cidade do nordeste - Maranhão. Rev Ciênc Saúde 2010; 12(1):45-48.

23. Thuler LCS, Bergmann A, Casado L. Perfil das Pacientes com Câncer do Colo do Útero no Brasil, 2000-2009: Estudo de Base Secundária. Rev Brasileira de Cancerologia 2012; 58(3):351-357.

24. Oliveira AF, Cunha CLF, Viégas IF, Figueiredo IS, Brito LMO, Chein MBC. Estudo sobre a adesão ao exame citopatológico de papanicolau em um grupo de mulheres. Rev Pesq Saúde 2010; 11(1):32-37.

25. Machado MS, Cantanhede KL, Leal PC, Nascimento MDSB, Silva RR, Bezerra JM. Estudo Retrospectivo do Câncer de Colo do Útero das Pacientes Atendidas no Hospital de Base do Distrito Federal. NewsLab 2005; 73:82-89.

26. Brasil. Ministério da Saúde (MS). Secretaria de Atenção à Saúde. Instituto Nacional de Câncer (Inca). Coordenação de Prevenção e Vigilância. Nomenclatura brasileira para laudos cervicais e condutas preconizadas: recomendações para profissionais de saúde. Rio de Janeiro: Inca; 2006. p. 65. 
27. Lima TM, Castelo ARP, Oliveira RG, Costa LQ, Tavares MC, Santiago JMV, Pinheiro AKB. Analysis of the cytopathological reports achieved and not removed from a Centro de Parto Natural (CPN) in Fortaleza-Ce: a documentary study. Online Brazilian Journal of Nursing 2009; 8(2):1-9.

28. Brasil. Ministério da Saúde (MS). Sistema de Informação do câncer do colo do útero e Sistema de Informação do câncer de mama. 2009. [Internet]. [acessado $2010 \mathrm{dez} 21$ ]. Disponível em: http:// w3.datasus.gov.br/siscam/index.php?area $=0401$

29. Casarin MR, Piccoli JCE. Educação em saúde para prevenção do câncer de colo do útero em mulheres do município de Santo Ângelo/RS. Cien Saude Colet 2011; 16(9):3925-3932.

30. Leinonen MK, Nieminen P, Lonnberg S, Malila N, Hakama M, Pokhrel A, Laurila P, Tarkkanen J, Anttila A. Detection rates of precancerous and cancerous cervical lesions within one screening round of primary human papillomavirus DNA testing: prospective randomised trial in Finland. BMJ 2012; 345:e7789.

31. Boone JD, Erickson BK, Huh WK. New insights into cervical cancer screening. J Gynecol Oncol 2012; 23(4):282-287.

32. Lorincz AT, Richart RM. Human papillomavirus DNA testing as an adjunct to cytology in cervical screening programs. Arch Pathol Lab Med 2003; 127(8):959-968.

33. Homma A, Moreira M. Novos desafios para capacitação tecnológica nacional de vacinas: inovação tecnológica autóctone e transferência de tecnologia. Cad Saude Publica 2008; 24(2):238-239.

34. Ribassin-Majed L, Lounes R, Clemencon S. Efficacy of vaccination against HPV infections to prevent cervical cancer in France: present assessment and pathways to improve vaccination policies. PLoS One 2012; 7(3):e32251.

35. Andrade JMd. Limitações para o sucesso do rastreamento do câncer de colo no Brasil. Rev Brasileira de Ginecologia e Obstetrícia 2012; 34(6):245-247.

36. Vale DBAP, Morais SS, Pimenta AL, Zeferino LC. Avaliação do rastreamento do câncer do colo do útero na Estratégia Saúde da Família no Município de Amparo, São Paulo, Brasil. Cad Saude Publica 2010; 26(2):383-390.

37. Brasil. Ministério da Saúde (MS). Portaria no 531, de 26 de março de 2012. Institui o Programa Nacional de Qualidade em Mamografia (PNQM). Diário Oficial da União 2012; 27 mar.

Artigo apresentado em 15/03/2013

Aprovado em 27/04/2013

Versão final apresentada em 01/05/2013 